Conclusions: ( ${ }^{1}$ Shedding of the Fc receptor CD16 has an unexpectedly complex impact on NK cell responses. Shedding this receptor renders NK cells less potent at CD16-mediated activation, as expected, but promotes the detachment from opsonized targets to aid sequential target cell surveillance. Thus, counter-intuitively, shedding of Fc receptor CD16 can positively impact immune responses. ${ }^{2}$ Genetic variation modulates the nanoscale organisation of inhibitory NK cell receptors, which in turn impacts receptor signalling. This may be important in how genetic variation impacts immune responses and disease susceptibilities.

Disclosure of Interest: None declared

DOI: 10.1136/annrheumdis-2018-eular.7694

\section{SP0095 INFLAMMATORY CYTOKINES AS KEY MESSENGERS OF THE IMMUNE SYSTEM}

M.I. Koenders. Experimental Rheumatology, Radboud university medical center, Nijmegen, Netherlands

Intercellular communication is mediated by direct cell contact as well as via soluble factors like cytokines. Cytokines are signalling proteins that bind to a receptor on a recipient cell, thereby activating an intracellular signalling pathway. This in turn may affect various biological functions like activation, differentiation or proliferation of the cell.

In this lecture, the role of inflammatory cytokines as key messengers of the immune system will be discussed. Targeting of hallmark cytokines in rheumatic diseases, like TNF, IL-6, GM-CSF, and IL-17, has changed the therapeutic landscape, and novel pro-inflammatory cytokines are currently under investigation as therapeutic targets. Furthermore, anti-inflammatory cytokines may be applied as therapeutic agents.

Cytokines act on the innate and adaptive immune system, as well as on nonimmune cells. How exactly do these key messengers control our immune system in health and disease?

To unravel the cytokine network that drives the dysregulated immune system in rheumatic diseases, findings from in vitro studies, animal experiments and clinical trials will be combined.

Disclosure of Interest: None declared

DOI: 10.1136/annrheumdis-2018-eular.7846

\section{FRIDAY, 15 JUNE 2018}

\section{WIN and HOT session}

\section{SP0096 ADVANCES IN THE MANAGEMENT OF OSTEOPOROSIS}

\section{Roux. Rhumatologie, Hôpital Cochin, Paris, France}

The number of fractures related to osteoporosis is expected to increase in the next few decades, because of the increasing number of frail elderly patients at high risk of falls and fractures. Guidelines are available worldwide for the screening of patients at high risk of fracture, and for appropriate treatment including non pharmacological treatments for falls prevention: A number of questions will be discussed during the session. Why should we treat? Because the burden of osteoporosis is related to fractures, and their consequences on morbidity, and even on mortality for the more severe ones. When should we treat? There is an immediate increase of sustaining a new fracture in the 2-3 years following a first one; this represents a unique window of opportunity for treatment. How should we treat? Treatments are effective in prevention of fracture, and re fracture, providing that we use the more effective treatment, with the appropriate sequences. How long should we treat? The treat to target strategy must be used in patients having a low bone mineral density, and obtaining a T score above -2 is a reachable target. A current challenge is the patients' view on side effects of the treatments; although they are very rare, their perception is very high. A number of qualitative studies sought to assess patients' fears and beliefs and provide wordings to clarify the fracture risk concept. The prevention of fragility fractures is now within our reach.

Disclosure of Interest: None declared

DOI: 10.1136/annrheumdis-2018-eular.7809

\section{SP0097 CHECK-POINT INHIBITORS: WHAT ARE THEY USED FOR, ABOUT SIDE-EFFECTS AND PREDICTION OF EFFICACY}

\section{E. Kapiteijn. Medical Oncology, LUMC, Leiden, Netherlands}

The development of immune checkpoint inhibitors targeting cytotoxic T-lymphocyte antigen 4 (CTLA-4), programmed cell death-1 (PD-1) and programmed death-ligand 1 (PD-L1) have significantly improved the treatment of a variety of metastasized cancers, such as melanoma, lung cancer, renal and bladder cancer. Immune checkpoint inhibitors enhance antitumor immunity by blocking negative regulators of T-cell function that exist both on immune cells and on tumour cells. Although these agents can lead to remarkable responses, their use can also be associated with unique immune-related adverse effects (irAEs).

Consensus guidelines regarding the treatment of the most common irAEs includ ing rash, colitis, hepatitis, endocrinopathies, and pneumonitis have been established. The mainstay of irAE treatment consists of immunosuppression with corticosteroids or other immunosuppressant agents such as infliximab; most irAEs will resolve with appropriate management.

Nevertheless, a substantial proportion of patients treated with checkpoint inhibitors do not respond. Several markers have shown promising predictive value, including baseline and post-treatment changes in leucocyte counts, lactate dehy drogenase and C-reactive protein. To date, research on routinely available blood, biopsies and clinical markers has focused primarily on checkpoint inhibitors use in melanoma and lung cancer, but preliminary evidence is emerging for other cancer types.

Disclosure of Interest: None declared

DOI: 10.1136/annrheumdis-2018-eular.7786

\section{SP0098 \\ HOT SESSION: IMMUNE RELATED ADVERSE EVENTS FROM CHECKPOINT INHIBITOR THERAPY}

\section{Calabrese, on behalf of IRaeS eular. Rheumatology, Cleveland Clinic, -} Cleveland, USA

Advances in cancer immunotherapy have made it now one of the cornerstones of care for patients with malignancies. Much of these advances have come through the development of checkpoint inhibitors which target negative regulatory signals in the adaptive immune response leading to re-invigoration of cell mediated immunity which had become suppressed or exhausted within the tumoral environment. With such reinvigorated immune response a collateral effect has been the development of autoimmune and or autoinflammatory complications (known as immune related adverse events or irAEs) seen in virtually every organ system. Among these are rheumatic complications estimated to observe in $4 \%-7 \%$ of the exposed population and often requiring acute and chronic immunosuppression to adequately manage. Numerous rheumatic irAEs have been reported the most common of which is inflammatory arthritis as well as myositis, PMR, vasculitis and sicca and others. Rheumatologists are now increasingly seeing such patients as the proliferation of checkpoint therapy increases (three are estimated to be several hundred thousand patients on such therapy in the US with another 100000 patients in varying clinical trial of cancer immunotherapeutics). irAEs in genera have been posited to be the potential Achilles heel of cancer immunotherapy. Rheumatologist will be increasing called upon to take key roles in the management of irAEs with a broad spectrum of immunosuppressive and targeted therapies.

Disclosure of Interest: None declared

DOI: 10.1136/annrheumdis-2018-eular.7762

\section{FRIDAY, 15 JUNE 2018 \\ Challenges in systemic lupus diagnosis}

\section{SP0099 A CASE OF PAINFUL DYSPNEA}

\section{Gatto. Unit of Rheumatology, University of Padova, Padova, Italy}

A 20-year-old female patient was admitted to ER due to dyspnea and stinging chest and interscapular pain, associated with neck pain worsened by physical exercise.

She reported worsening of respiratory symptoms in the past ten days and concomitant onset of acrocyanosis. At physical examination she displayed tachycardia and tachypnea, no abnormalities at lung auscultation, with oxygen saturation $94 \%$ at pulse oxymeter. Arterial blood gas tests showed a decreased pO2 and pCO2. Urgent blood examination showed low leucocyte count 2900/mm3 and mild normocytic anaemia $\mathrm{Hb} 10.8 \mathrm{~g} / \mathrm{dl}$. Due to chest pain, dyspnea and concomitant gas findings, a CT scan was prompted in the suspicion of pulmonary embolism, yet no vascular abnormalities were shown, but a small rim of pleural fluid at the right base.

The patient was admitted to Internal medicine department where she underwent pulmonary function tests showing a restrictive respiratory pattern with decreased TLCO and but normal KCO. HRCT was performed ruling out interstitial lung disease. Elevation of right emidiaphgram was shown at AP imaging. 